\title{
GESTION DEL ÉXITO ORGANIZACIONAL COMO ESTRATEGIA DE VALOR PARA MINIMIZAR LA RELUCTANCIA EN PYMES ${ }^{1}$
}

\author{
ORGANIZATIONAL MANAGEMENT AS SUCCESS STRATEGY \\ VALUE TO MINIMIZE RELUCTANCE SMES
}

\author{
Ramiro Gamboa Suárez $z^{2}$ Luis Alfredo Jiménez Rodriguez ${ }^{1}$ \\ Universidad Corhuila-Colombia \\ Universidad Francisco de Paula Santander-Ocaña, Colombia
}

RECIBIDO: Septiembre 02 de 2015

ACEPTADO: Noviembre 13 de 2015

DOI

\begin{abstract}
RESUMEN
El propósito esencial de la investigación, fue analizar la gestión del éxito organizacional como herramienta de valor para minimizar la reluctancia en Pymes. Metodológicamente, se circunscribió dentro del paradigma post-positivista, enfoque epistemológico cualitativo, se aplicó un enfoque introspectivo vivencial, se hizo uso de la observación, lo cual implica la inserción del hecho en el marco referencial de valores, creencias y actitudes, constituyendo una red de significados personales. Se asumió la teoría fundamentada, ya que permite la integración objeto-sujeto y responde al problema investigativo en el área social. Se utilizo la entrevista no estructurada, la cual fue aplicada en organizaciones seleccionadas, previa definición de los informantes clave, los cuales se convirtieron en fuente de información. Los resultados del presente trabajo, concluyeron que aspectos tales como el soporte estratégico para fortalecer la conciencia empresarial, la arquitectura del aprendizaje organizacional, la opción de crecimiento para mejorar el ambiente organizacional y de compromiso, y la construcción de sugerencias por parte del activo humano como estrategia empresarial, permitirán, el aprovechamiento de los hallazgos como base expedita para que se conozcan las realidades de la gestión del éxito organizacional como herramienta de valor para minimizar la reluctancia en Pymes.
\end{abstract}

Palabras clave: Éxito Organizacional, Gestión, Reluctancia, Pymes.

\begin{abstract}
The essential purpose of the research was to analyze the management of organizational success as a valuable tool to minimize the reluctance in SMEs. Methodologically, is confined within the post-positivist paradigm, qualitative epistemological approach, an experiential introspective approach was applied, use was made of the observation, which involves the insertion of fact in the referential framework of values, beliefs and attitudes, constituting a network personal meanings. grounded theory was assumed, allowing object-subject integration and responds to the research problem in the social area. Unstructured interview was used, which was applied to selected organizations, predetermination of key informants, which became a source of information. The results of this study concluded that aspects such as strategic to strengthen corporate awareness support, the architecture of organizational learning, growth option to improve organizational and commitment environment, and building suggestions by the human asset as business strategy, allow the use of findings as expeditious basis for the realities of managing organizational success as a valuable tool to minimize the reluctance SMEs are known.
\end{abstract}

Key words: Organizational Success, Management, Reluctance, SMEs.

Este artículo se puede referenciar

Gamboa Suárez, R. \& Jimenez Rodriguez, L(2016). Gestion del éxito organizacional como estrategia de valor para minimizar la reluctancia en pymes.

En Desarrollo Gerencial Revista de la Facultad de Ciencias Económicas Administrativas y Contables de la Universidad Simón Bolivar-Colombia, 8(1), 123-142.

1 Este artículo constituye un avance del proyecto de investigación "Gestion del éxito organizacional como estrategia de valor para minimizar la reluctancia en pymes".

2 Docente-Investigador. Universidad Corhuila-Comombia. Email: gazur11@yahoo.com 


\section{Introducción}

Las organizaciones, a través de los tiempos han ido evolucionando, esto gracias a la adquisición de mayor experiencia, trasegando hacia el desarrollo de mejores o nuevas tecnologías, capacitan a sus trabajadores, entre otros aspectos; persiguiendo de esta manera objetivos direccionados a incrementar la productividad, el rendimiento de las operaciones, la rentabilidad económica y financiera; que en términos administrativos se enfocan al mejoramiento continuo de los procesos, productos o servicios, obteniendo así mayores ingresos y minimizando riesgos, lo cual propicia la eficiencia y eficacia de las operaciones, convirtiéndose en organizaciones socialmente responsables, globalizadas, con estándares orientados al logro de excelencia organizacional, siendo competitivos y competentes en las actividades emprendidas.

Desde esa perspectiva, las organizaciones deben involucrar en el logro de objetivos y metas a los grupos de interés propiciando de esta manera la ejecución de acciones concretas, por tal motivo, la interacción de emprendimientos genera sinergia que transforma realidades y permite evolucionar agregando valor a la empresa bajo condiciones de convivencia ajustadas a cada propósito en forma coordinada y armónica.

Lo descrito conlleva a considerar los principios de la gestión organizacional cuya aplicabilidad en prospectiva es condición para el logro de procesos que incorporen mejoramiento continuo en la calidad de los productos y servicios, aplicando para ello métodos ajustados a las condiciones de cada entidad, aunque las herramientas para el control de gestión no necesariamente reducen las ineficiencias en los procesos y procedimientos de manera espontánea, su aplicación debe considerarse para lograr que los clientes no resulten afectados por inconvenientes internos de la organización. Entonces, los aspectos referidos al mejoramiento continuo de la calidad afectan el funcionamiento de las organizaciones, coartando de esta manera el desarrollo integral de estas.

En todo caso, las condiciones presentes en los procesos, procedimientos y actividades que no permiten el desarrollo eficiente y eficaz de la organización, requieren de un plan de mejoramiento continuo que conduzca a la reducción de la incertidumbre relacionada con la calidad y el posicionamiento de la empresa en su entorno. El estudio englobará áreas de relevancia en las Pymes donde se gesten procesos reluctantes, identificando a su vez aspectos inmateriales que subyacen en las decisiones de los participantes Summers (2006), cuyos factores no propician adecuadamente la gestión organizacional.

Es así, como en las Pymes (Pequeñas y Medianas Empresas), a nivel mundial se integran sectores económicos de diversa índole, desde las empresas familiares, donde las decisiones normalmente son asumidas por los padres como representantes del núcleo familiar o por sus descendientes cuando se transfieren en 
forma escalonada el proceso de gestión, complicando en ocasiones el desarrollo de las operaciones, cuyo efecto se evidencia en acciones confusas que al final no generan valor agregado en las organizaciones.

Por lo anterior mencionado, parte de esas decisiones son asumidas desde el punto de vista emocional de cada individuo, descartando la coherencia requerida en los procesos tecnológicos y científicos, cuyo efecto es el desconocimiento de métodos o técnicas relevantes en su cuantía e importancia que contribuyan a mejoramiento continuo de la calidad en los productos y servicios, por ello la renuencia o reluctancia en el reconocimiento de los avances tecnológicos y científicos se reflejan en la ingobernabilidad de las entidades cuyos procesos gerenciales son ambiguos y de difícil entendimiento y desarrollo.

El término reluctancia en el campo de los comportamientos organizacionales, es un proceso cuyo efecto en los costos de producción de un bien o servicio o incidir en los gastos administrativos o financieros por lo que se requiere identificarlos y evaluarlos en cada uno de los procesos; así como valorarlos para determinar la incidencia sobre los resultados para eliminarlos o minimizarlos con el fin de hacer las operaciones cada vez más eficientes orientando la mejora continua y evitando así el proceso reluctante que impide logros en las acciones gerenciales propuestas, aduciendo Gutiérrez (2012), que en su filosofía el sistema de administración no es difícil comprenderlo, pues se caracteriza por el profundo sentido humano y común, entonces, la gestión administrativa consiste en realizar las labores pertinentes, sin complejidades, pero eso sí, dentro de la rigurosidad que enmarca el gerenciamiento moderno.

Dentro de los factores considerar en el proceso administrativo y determinante en el proceso reluctante es relevante considerar la comunicación entre los individuos que integran el circuito de acciones y decisiones. Cada forma de comunicación a través de cartas, anuncios, emisiones escritas y radiodifundidas lleva implícito un mensaje que debe ser preparado y medido en cuanto a sus efectos en los receptores ya que la reacción puede ocasionar reluctancia en las acciones anunciadas Peiffer (1994), quien habla del pensamiento positivo de manera clara e ilustrativa, con enseñanzas de casos reales aplicables, y define la comunicación como el elemento base del proceso gerencial.

En efecto, el proceso comunicativo debe incorporar condiciones y reglas para el desarrollo y mantenimiento de las relaciones organizacionales que impidan el surgimiento de mensajes estériles y sin sentido común, lo cual suma complejidad a las decisiones generando como consecuencia pérdida de valor en la organización por la toma de decisiones sesgadas, amañadas y reluctantes en el proceso administrativo, creando caos, además desorden y falta de motivación de forma generalizada.

En este orden de ideas, la gestión del éxito organizacional como estrategia de valor para minimizar la 
reluctancia en las Pymes, se fundamenta en hechos que se gestan en la realidad empresarial y de los cuales no hay evidencia de su existencia solo se perciben las consecuencias económicas y financieras, este aspecto como factor clave de la gestión en las organizaciones propicia acciones decisorias en los directivos. Este hecho inmaterial en su forma debe ser gestionado por personas, quienes con sus decisiones podrán valorizar o desvalorizar a la organización, debe por sobre todo ser impermeable, blindado en cuanto a la posibilidad de contaminación, generando así valor intangible a las organizaciones.

Es así, como el talento humano constituye uno de los factores determinantes para la obtención de valor agregado, dadas sus decisiones. Este valor se potencia cuando el conocimiento se coloca en función del logro de los objetivos de la organización. Carvajal (2002), esgrime que del capital humano depende en gran medida de la capacidad de las organizaciones para desarrollar y aprovechar el conocimiento.

En ese sentido, se comprende, que el talento humano involucra conocimientos, habilidades, valores y el potencial innovador que requiere la organización. La gestión de las condiciones donde actúan estas personas requiere de atención especial, suponiendo capacidad de los directivos para identificar, medir, desarrollar y renovar el factor humano para el futuro éxito de la organización. El talento humano reside en los individuos, quien con su apreciación, conocimiento y gestión aportan valor a la organización, evitando de esta manera pérdidas asociadas al tiempo improductivo y acciones desviadas de los objetivos y metas planteadas.

Es así como los procesos requieren acción directa del ser humano para minimizar el proceso de reluctancia que por analogía se pudiese presentar, lo cual debe ser considerado para lograr el mejoramiento continuo de la calidad en los bienes y servicios, propendiendo así mayor eficiencia y eficacia en la interacción de las acciones decisorias en la organización.La globalización de la información conlleva a considerar que toda acción emprendida debe estar documentada y demostrada su coherencia con los retos y objetivos propuestos lo cual permite gestionar los recursos disponibles no utilizados e inferir, que hay necesidad de formular decisiones, reconocer debilidades en los procesos, pero ante todo entender cuál es la vía más expedita para lograr empoderamiento progresivo de las Pymes que permitan agregar valor a las organizaciones y en consecuencia a los países.

Al respecto, Segui (2007), señala que la realidad económica de Colombia ha permitido que las Pymes en especial, reconozcan dentro de su operación el efecto de los intangibles dentro de la cadena de valor de sus bienes y servicios, tornándose mucho más compleja su valoración. Este planteamiento es apoyado por Sapag (2008), quien refiere que los efectos intangibles constituyen los de mayor dificultad de cuantificar, por su carácter de inmedibles, naturaleza antagónica, algunos favorables otros desfavorables. 
Lo antes descrito, ha favorecido la aparición de diferentes metodologías, entre ellas el proceso de excelencia empresarial y calidad en el servicio Peters y Waterman (1986), el esquema de las 7 S de Mckinsey, establece que las decisiones permiten el sostenimiento en el mercado el cual se debe sustentar en la innovación constante, y no basarse en la estructura o estrategia como medios para lograr la eficiencia y productividad de la organización, sino que además consideran las capacidades de su personal y el estilo de liderazgo como idea orientadora, y el staff reducido como estilo de gestión, según sostiene Münch (2007).

Lo enunciado, constituye el fundamento para que las organizaciones en especial las Pymes, asuman conscientemente las deficiencias en procesos, procedimientos, técnicas o métodos presentes en el proceso administrativo, bajo la perspectiva de la reluctancia al considerar aspectos trascendentales, en especial lo relacionado con situaciones complejas, originadas de conductas reluctantes o renuentes de forma generalizada en estas organizaciones al no aceptar y reconocer la existencia de aspectos aunque pequeños o intangibles como factores que impiden generar valor y su consecuente potenciamiento hacia el éxito, en consecuencia emprender soluciones a problemas implica asumir complejidad en los seres humanos responsables, generando así retraso en la acciones generadoras de valor para la organización.

En atención a la problemática expuesta, CONPES (2013), expone que, la dinámica de las organizaciones en Colombia durante los últimos años, pese a las vicisitudes propias del entorno, ha venido enfocándose en el logro de un desempeño superior y una ventaja competitiva sostenida, debido a diversos factores tales como la liberalización generalizada de los mercados, así como ampliación de los mercados de capitales, cuyo ámbito de operación rivaliza y concurre en sectores productivos análogos de diferentes países. El desempeño superior suele referirse a logros frente a otras empresas similares en el mismo tipo de negocio y rivales, alcanzando objetivos en condiciones favorables.

En términos generales, la rentabilidad es una medida idónea del desempeño superior, asociada con el rendimiento del capital invertido en la empresa. Sin embargo, la rentabilidad puede resultar limitada para describir esfuerzos de organizaciones. El desempeño en términos de rentabilidad es una ventaja competitiva cuando la medición de los resultados es mayor al promedio de las empresas que compiten en un mismo mercado o conforman sector comercial o industrial.Dentro del marco jurídico colombiano y estadísticas publicadas por CONPES (2012), se evidencian dificultades que impiden a las Pymes lograr la competitividad deseada, dicha institución describe doce elementos sobre los cuales se fija la política de estado:

Poca sofisticación y baja agregación de valor en los procesos productivos, baja productividad y capacidad de generación de empleo en los sectores formales, baja productividad del sector agropecuario, altos 
niveles de informalidad empresarial y laboral, bajos niveles de innovación y absorción de tecnologías, poca profundidad y sofisticación del mercado financiero, deficiencias en la infraestructura de transporte y energía, baja calidad y poca pertinencia de la educación, estructura tributaria poco amigable a la competitividad, rezago en penetración de tecnologías de información y conectividad, degradación ambiental como limitante de la competitividad, debilidad de la institucionalidad relacionada con la competitividad.

Las Pymes colombianas, aportan a la producción interna compensación ante la caída de las exportaciones, generadas por la baja de materias primas como petróleo, dichas empresas han logrado llegar con sus productos a destinos cada vez más lejanos. (El tiempo, enero 19 de 2015). Las Pymes en la economía nacional y regional del Departamento del Huila, han permitido un viraje a la economía del país por ello es necesario proponer modelos que permitan su consolidación y pleno desarrollo.

Dentro de este orden de ideas, se hace necesario definir parámetros en el estudio, para adecuar la transformación de las Pymes hacia una gestión organizacional, en función a las circunstancias situacionales y coyunturales de cada región, para convertirlas en empresas generadoras de valor cuyos procesos reluctantes sean efectivamente conocidos, propiciando de este modo cambios estratégicos cuya consecuencia se traduzca en competitividad a través de la mejora continua de los procesos. Por tanto es pertinente cuestionar esta situación y emerger una interrogante que promulgue un proceso investigativo a efectos de responder: ¿Cómo es la gestión del éxito organizacional como estrategia de valor para minimizar la reluctancia en Pymes?

De lo anterior se desprende el objeto de este estudio basado en el análisis de la gestión del éxito organizacional como estrategia de valor para minimizar la reluctancia en Pymes; el cual se justifica ante las razones de que las Pymes son entidades generadoras de empleos e ingresos para los países, en este entorno se vislumbran factores claves que deben ser considerados para ser analizados en función de identificar los procesos débiles y la adecuación de su operación a las condiciones fluctuantes de los mercados, para ello es necesario considerar las actividades organizacionales, identificando aquellos procesos de gestión asociados a las personas cuyas acciones emprendidas son consideradas reluctantes e impiden a este tipo de organizaciones ser exitosas al no generar agregados de valor y en consecuencia rentabilidad económica.

Por lo que las Pymes, requieren mejorar la competitividad de sus productos y asegurar una mayor satisfacción de sus clientes, lo cual se logra, en gran parte, con un liderazgo a bajo costo, Porter (1980). Esta investigación contribuirá a identificar los procesos reluctantes, las actividades asociadas al mejoramiento continuo de la calidad, y por ende, identificar los procesos generadores de estas acciones, los cuales son 
susceptibles de un plan de mejoramiento, con el fin de reducirlos e incorporarlos al proceso de control administrativo, propiciando de este modo la competitividad y contribuyendo a mejorar la calidad de vida de los empleados, socios, y la cadena de empleos indirectos que generan por su actividad.

Esta investigación estableció una línea investigativa desde los sustentos teóricos que esbozados de manera subsecuente, los cuales tuvieron como propósito esgrimir y apoyar los preceptos y elementos conceptuales ligados a comprender la gestión del éxito organizacional como estrategia de valor para minimizar la reluctancia en pymes del municipio de Neiva, Huila, Colombia, los cuales son fundamentales para que estas organizaciones se acobijen con la gestión del éxito y su competitividad. De manera que su estado del arte se circunscribió a definir y plasmar los variados tópicos, guías, procesos y fases de la actual gestión del éxito organizacional como herramienta para minimizar la reluctancia en las Pyme.

De igual manera se estableció un recorrido metodológico con una carga epistémica atendiendo a la actual coyuntura de apertura hacia el conocimiento y su generación requerida por la multiplicidad de condiciones, situaciones y latitudes, de la humanidad, han cobrado importancia las investigaciones cuyo centro de atención son las subjetividades de los sujetos en su vida cotidiana. Dentro de este marco, para Berger y Luckman (1994), la realidad de la vida cotidiana se organiza alrededor del 'aquí' de mi cuerpo y el 'ahora' de mi presente. Este 'aquí y ahora' es el foco de la atención que presto a la realidad de la vida cotidiana. Llevando a entender el hecho investigativo en el campo donde operan quienes aportarán la información necesaria para la investigación, justifican el uso de técnicas de observación participante basadas en un guion de entrevista no estructurada. Flick (2012), ubica como métodos para la obtención de datos la observación y entrevistas.

Por lo antes expuesto se entiende que el complejo recorrido metodológico obedeció a un enfoque postpositivista, desde la teoría fundamentada como enfoque de la investigación ha venido desarrollándose desde que Strauss y Corbin(2002), la refirió como base para conocer profundamente él, o, los fenómenos sociales bajo su enfoque pragmático cita que los conceptos, a través de los cuales se formulan las creencias humanas son construcciones provisionales y lo verdaderamente relevante en las personas es la reconstrucción de sus prácticas morales, sociales y sus creencias. La tendencia más representativa y seguida es la de Strauss y Corbin (2002), para alcanzar el desarrollo de una teoría emergente a partir de la data. En este recorrido investigativo se seleccionaron unos informantes clave, bajo el criterio de ser: (a) visionarios, responsables y capacitadores y (b) comprometidos, analistas y proyectistas; utilizando las técnicas de observación participante y la entrevista semiestructurada, con recurrencia a la triangulación de datos, bajo el supuesto del empleo de distintas estrategias de recolección de datos. Su objetivo es verificar las tendencias detectadas en un determinado grupo de observaciones; cuya consideración ética es el abordaje de de la ciencia desde varias aristas es permisible que su meta sea resolver un problema o tratar de comprender algo. 


\section{Teorías de entrada}

\section{Gestión del éxito organizacional como estrategia de valor}

Para Fantova (2005), la gestión, es la rendición de cuentas ante el abanico de agentes interesados por los efectos que se espera que el proceso desencadene. Por tal, es fundamental la aprehensión de su significado para encasillar su derrotero hacia la gerencia capaz de mover esfuerzos con mira al logro de los resultados. La gestión es normalmente entendida como concepto de administración, o dirección de una organización cuyo objeto primordial es la consecución de resultados óptimos del negocio o compañía.

Para el logro de los resultados, la gestión se fundamenta en cuatro premisas básicas: (a) Entendimiento y reconocimiento de la estrategia, es decir; los pasos necesarios e indispensables para consolidar las acciones y hacerlas efectivas. (b) La cultura organizacional, entendida como el conjunto de postulados para hacer conocibles y alcanzables los valores corporativos. (c) La estructura, entendida como la linealidad y apertura hacia la entrada de conocimientos, y la posibilidad de establecer alianzas. (d) La ejecución, que nos lleva a tomar las decisiones acertadas hacia mayor productividad y mejora contínua.

Ahora bien, el éxito de manera indudable no puede ser fruto del azar, más bien, es el resultado de un marco sopesado, concienzudo y definido bajo los objetivos claros, medibles y alcanzables que propicien una acción posterior hacia su ejecución satisfactoria. Tal como describe Sánchez (2004), el éxito de una misión es la adecuación de los resultados a los objetivos, de forma estable, obteniendo un valor añadido neto alto.

Diagrama 1. Diagrama de Éxito

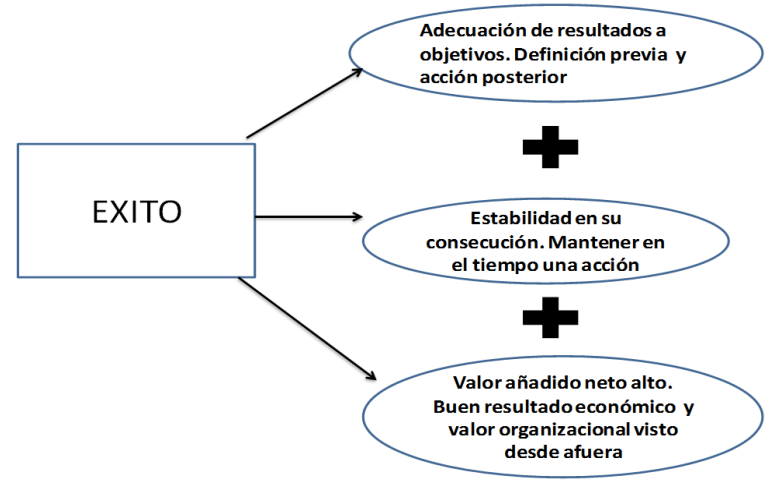

Fuente: Sánchez E (2004).

El fundamento esencial hacia el éxito en los negocios u organizaciones, está constituido por sustentar una fuerte cultura de trabajo, tal como lo expone Rohman (2014), en tal sentido la organización moderna 
deberá proveer recursos para dicha inversión, en la cual incorpore valores colectivos, aliente la satisfacción en el trabajo y promueva espacios de amplia espiritualidad laboral. Esto implica rodearse de personal idóneo, capacitado y con elevados estándares sociales. Pero, también la organización debe incentivar el acceso y promover su cultura hacia el logro social de sus metas.

Cada organización es una unidad independiente que posee sus propios derroteros y valores, estos últimos de manera general han sido legados por sus dueños, que a la vez son irradiados a todo el ente económico en pro de lograr el futuro adecuado. Los valores de la organización, aunque diferentes a los de sus dueños y colaboradores, deben ser aprehendidos y apropiados por todos sin excepción, como herramienta hacia el éxito organizacional.

\section{Reluctancia}

El investigador, se arriesga, sin querer entablar juicios de valor a definir la reluctancia desde el punto de vista gerencial como: "Situación donde la gerencia pospone las decisiones y es reacia a tomarlas por condiciones internas o externas y que entorpecen la adecuada gestión organizacional. Es un proceso de rechazo en el que se involucra la administración, siendo entonces oponible a la adecuada toma de decisiones. Adicional nos daría la postura de ser una acción, donde las decisiones son pospuestas o más bien se actúa con miedo, displicencia, dificultad o simplemente por falta de claridad y compromiso".

\section{Método}

El abordaje metodológico de la investigación se fundamentará en desarrollar la aplicación del enfoque cualitativo, el cual está relacionado con la inducción analítica bajo el paradigma pos-positivista y la corriente filosófica del idealismo, para el desarrollo del objeto de la investigación. Ahora bien, el enfoque cualitativo según Bernal (2006) adaptado por Pelekais et al, (2011), se orienta a profundizar casos específicos y no se generaliza. Se preocupa no por medir, sino, cualificar y describir el fenómeno social a partir de rasgos determinantes, según sean percibidos por los elementos mismos que están dentro del objeto de estudio.

En la actualidad, según Anguera (2008), la investigación cualitativa sigue desarrollándose y enriqueciéndose. Se usa en la antropología, ciencias sociales, psicología, la justicia, organizaciones, comunicaciones incluso en medicina, enfermería y trabajo social. Además, Denzin y Lincon (2012), indican que la investigación cualitativa implica un enfoque interpretativo y naturalista del mundo, lo cual significa que los investigadores cualitativos estudian las cosas en sus escenarios naturales, tratando de entender o interpretar los fenómenos en función de los significados que las personas le dan. Por tanto, lo importante es demostrar 
la interacción entre el individuo y la situación investigativa, estando entonces cubierta por el tamiz de la subjetividad.

En concordancia a lo descrito, lleva a entender el hecho investigativo en el campo donde operan quienes aportarán la información necesaria para la investigación, justifican el uso de técnicas de observación participante basadas en un guion de entrevista no estructurada. Flick (2012), ubica como métodos para la obtención de datos la observación y entrevistas.

Los participantes en la toma de la data se circunscribió a organizaciones Pymes, que abarco una muestra de 5 ellas y sus informantes clave, gerencial y administrativo. Por ser una investigación de tipo cualitativo los participantes fueron seleccionados de acuerdo a la experiencia del investigador y el conocimiento del medio donde se realizo la toma de la data. Para el presente desarrollo investigativo se definieron las organizaciones descritas en el acápite anterior en su número e informantes clave.

La técnica de obtención de la data se circunscribió a la entrevista semiestructurada, donde se desarrollo un guion y dada la condición de la teoría fundamentada aplicada se aproximo a entablar más conversaciones que surgieron a partir de la aplicación de las preguntas.

Como colofón al tratado del componente cualitativo de la investigación, según señalan Martínez (2010), dentro de la investigación cualitativa se conocen cuatro procesos cognitivos que aparecen inherentes al estudio: comprehensión, sintetización, teorización y recontextualización. Como resultado de lo anterior, el investigador cualitativo debe alcanzar un nivel razonable de comprensión antes de ser capaz de sintetizar, y no es posible teorizar antes de haber sintetizado, finalmente la recontextualización no puede tener lugar mientras los conceptos o modelos en la investigación no han sido desarrollados totalmente.

Respecto del desarrollo del modelo asumido por el investigador de la teoría fundamentada, pertinente para el abordaje de la investigación, se asumió la postura descrita por Strauss (1967), y respecto a las fases: codificación y categorización de la información, muestreo teórico y comparación constante. Cuando el investigador está inmerso dentro de la adopción por primera vez de una investigación de este tipo debe seguir el proceso descrito por Glaser y Strauss (1967), en cuanto a tener presente procedimientos para desarrollar la teoría fundamentada, los cuales se centran en el método de la comparación constante y el muestreo teórico, al abordar investigaciones de índole social.

En atención a lo anteriores importante considerar la rigurosidad en la aplicación del procedimiento utilizado, al finalizar el análisis, es permitido responder la premisa básica en cuanto a definir de que manera la 
gestión del éxito organizacional constituye una estrategia de valor válida para minimizar la reluctancia en las Pymes y alternativamente permite identificar el proceso de reluctancia presente en el entorno estudiado, el cual condiciona la toma de decisiones inapropiadas, siendo estos comprendidos en función a los factores reluctantes obstaculizadores del éxito organizacional en las Pymes.

\section{Resultados}

El desarrollo metodológico basado en la teoría fundamentada requiere realizar una triangulación de la data para definir las categorías, subcategorias y la saturación de las mismas, por ello se acudió al proceso de triangulación como se muestra en seguida

Cuadro 1. Triangulación

\begin{tabular}{|c|l|}
\hline Realidad & $\begin{array}{l}\text { La información que se suministró por parte de los informantes clave, está fundamentada por las } \\
\text { experiencias y percepciones de la realidad del entorno, éstas fueron sustraídas de las entrevistas } \\
\text { que se realizaron. }\end{array}$ \\
\hline Teoría Referencial & $\begin{array}{l}\text { La misma se deriva por los aportes de diversos autores, que suministran la información para las } \\
\text { categorías que han sido desarrolladas en la presente investigación, esto ha permitido profundizar } \\
\text { sobre el estudio contribuyendo al proceso de interpretación }\end{array}$ \\
\hline Aportes deI Investigador & $\begin{array}{l}\text { Esta surge de la Información experiencial del autor lo que permite conocer su punto de vista, } \\
\text { saberes y aportes de este. }\end{array}$ \\
\hline
\end{tabular}

Fuente: Elaboración propia (2016). 
Cuadro 2. Recopilación de la información y decodificación de datos

\begin{tabular}{|c|c|c|c|c|c|}
\hline Propósito & $\begin{array}{l}\text { Categorías } \\
\text { encontradas }\end{array}$ & $\begin{array}{l}\text { Teoría o } \\
\text { sistema } \\
\text { referencial }\end{array}$ & Realidad & $\begin{array}{l}\text { Categorías } \\
\text { emergentes }\end{array}$ & Aportes \\
\hline $\begin{array}{c}\text { Factores clave } \\
\text { para la gestión } \\
\text { del éxito } \\
\text { organizacional } \\
\text { en las Pymes. }\end{array}$ & $\begin{array}{c}\text { Factor Humano. } \\
\text { Autonomía. } \\
\text { Toma de } \\
\text { Decisiones. } \\
\text { Roles. } \\
\text { Cobertura de } \\
\text { riesgos. } \\
\text { Margen de } \\
\text { Comercialización. } \\
\text { Fidelización del } \\
\text { cliente. } \\
\text { Confianza en el } \\
\text { Mercado. } \\
\text { Calidad del } \\
\text { Servicio. } \\
\text { Formación. } \\
\text { Beneficios } \\
\text { emocionales. }\end{array}$ & $\begin{array}{l}\text { Campos, (2007). } \\
\text { Lara }(2004) . \\
\text { Sánchez (2004). } \\
\text { Alles }(2005) .\end{array}$ & $\begin{array}{c}\text { Talento humano como } \\
\text { factor integral. } \\
\text { Autonomía que cada } \\
\text { persona. } \\
\text { Los niveles gerenciales } \\
\text { establecen límites para } \\
\text { disponer la calidad de los } \\
\text { procesos. } \\
\text { Manejo de precios } \\
\text { coherentes con el } \\
\text { mercado para operar en } \\
\text { equilibrio con margen de } \\
\text { comercialización. } \\
\text { Formación del recurso } \\
\text { humano. } \\
\text { Plataforma filosófica por el } \\
\text { aprendizaje organizacional, } \\
\text { reconociendo las } \\
\text { experiencias vividas. } \\
\text { Estilo de gerencia de } \\
\text { pirámide invertida. }\end{array}$ & $\begin{array}{c}\text { Cobertura de } \\
\text { Riesgos. } \\
\text { Fidelización del } \\
\text { cliente. } \\
\text { Beneficios } \\
\text { emocionales. }\end{array}$ & 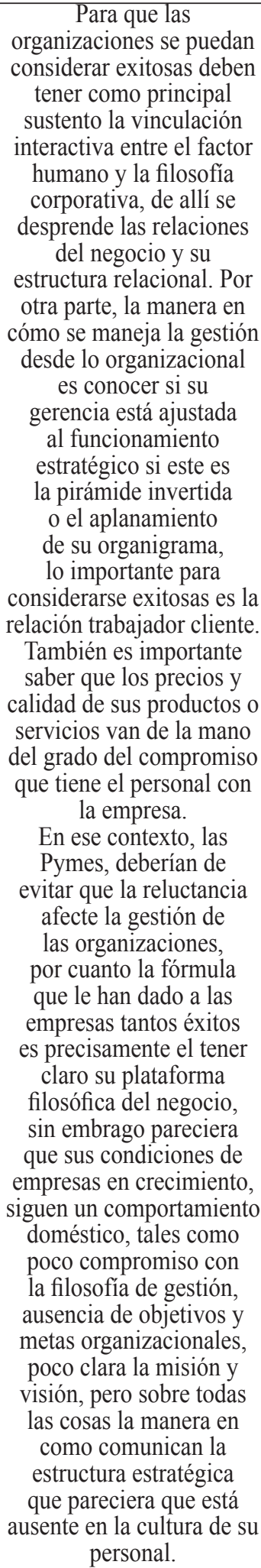 \\
\hline
\end{tabular}




\begin{tabular}{|c|c|c|c|c|c|}
\hline $\begin{array}{c}\text { Elementos } \\
\text { generadores } \\
\text { de la posición } \\
\text { competitiva } \\
\text { global en las } \\
\text { Pymes. }\end{array}$ & $\begin{array}{c}\text { Responsables de } \\
\text { los procesos } \\
\text { Rutas que guían } \\
\text { decisiones } \\
\text { Políticas Flexibles } \\
\text { Negociaciones } \\
\text { acordadas } \\
\text { Respaldo a crédito } \\
\text { Exportaciones de } \\
\text { productos } \\
\text { Regulaciones } \\
\text { legales } \\
\text { Seguimiento a } \\
\text { clientes } \\
\text { Calidad de } \\
\text { producto } \\
\text { Diferenciación de } \\
\text { productos } \\
\text { Planeación } \\
\text { estratégica en } \\
\text { equipos }\end{array}$ & $\begin{array}{l}\text { Fletcher (2010). } \\
\text { Alcaide (2015) }\end{array}$ & $\begin{array}{l}\text { Entendimiento de los } \\
\text { trabajadores por parte de } \\
\text { la empresa entendiendo } \\
\text { su responsabilidad en los } \\
\text { procesos y permitir tomar } \\
\text { decisiones. } \\
\text { Fidelidad de los clientes } \\
\text { por precios con una política } \\
\text { flexible de asignación. } \\
\text { Acciones hacia al buen } \\
\text { servicio, seguimiento } \\
\text { a clientes, calidad del } \\
\text { producto, procesamiento y } \\
\text { empaque. } \\
\text { Planeación estratégica } \\
\text { desarrollada por equipo } \\
\text { de trabajo y existe una } \\
\text { marcada diferenciación de } \\
\text { los productos. } \\
\text { Involucramiento de talento } \\
\text { humano en los diseños de } \\
\text { los productos. } \\
\text { Segmentación de la } \\
\text { fuerza de ventas y de sus } \\
\text { directores. } \\
\text { Estrategias de ventas que } \\
\text { permiten trazar el mapa de } \\
\text { ruta para la formación y } \\
\text { capacitación del personal. }\end{array}$ & $\begin{array}{c}\text { Regulaciones } \\
\text { legales } \\
\text { Políticas } \\
\text { organizacionales } \\
\text { flexibles }\end{array}$ & $\begin{array}{c}\text { Cuando se habla de } \\
\text { posición competitiva, } \\
\text { no hay que olvidar que } \\
\text { todo indica que hay que } \\
\text { establecer que el precio, } \\
\text { la calidad del servicio, } \\
\text { la fuerza de venta entre } \\
\text { otros, son los factores } \\
\text { fundamentales para medir } \\
\text { que tan competitiva } \\
\text { es la organización } \\
\text { en el mercado, para } \\
\text { ello también hay que } \\
\text { considerar las estrategias } \\
\text { de venta. La fidelización } \\
\text { del cliente no se logra con } \\
\text { el ofrecimiento de una } \\
\text { buena política de precios, } \\
\text { sino que también hay que } \\
\text { considerar la formación y } \\
\text { capacitación de la fuerza } \\
\text { de venta que permita } \\
\text { alcanzar competencias } \\
\text { que lo hagan más integral } \\
\text { a la hora de ofrecer los } \\
\text { productos o servicios. }\end{array}$ \\
\hline
\end{tabular}




\begin{tabular}{|c|c|c|c|c|c|}
\hline $\begin{array}{c}\text { Actividades } \\
\text { organizacionales } \\
\text { que determinan } \\
\text { a la reluctancia } \\
\text { en las Pymes. }\end{array}$ & $\begin{array}{c}\text { Asesoramiento a } \\
\text { los clientes } \\
\text { Consultas } \\
\text { constantes a la } \\
\text { gerencia } \\
\text { Reacción a } \\
\text { innovar } \\
\text { Salario emocional } \\
\text { Experimentando } \\
\text { cambios } \\
\text { Análisis de } \\
\text { problemas en } \\
\text { conjunto } \\
\text { Decisiones } \\
\text { centradas en la } \\
\text { gerencia } \\
\text { Filosofía de la } \\
\text { empresa } \\
\text { Cultura } \\
\text { institucional } \\
\text { Liderazgo } \\
\text { autocrático }\end{array}$ & $\begin{array}{c}\text { Kaplan y } \\
\text { Norton (2007). } \\
\text { David (2013). } \\
\text { Serna (2014). }\end{array}$ & 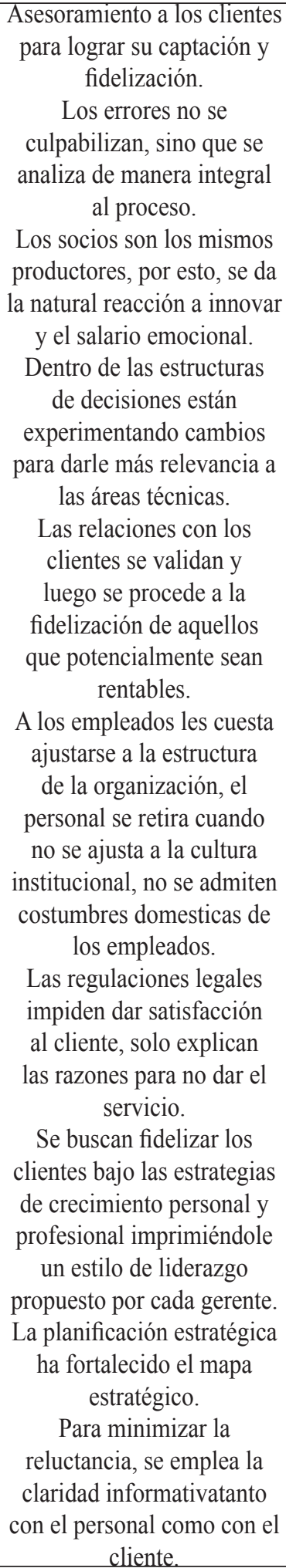 & $\begin{array}{c}\text { Filosofía } \\
\text { empresarial } \\
\text { Liderazgo } \\
\text { autocrático }\end{array}$ & 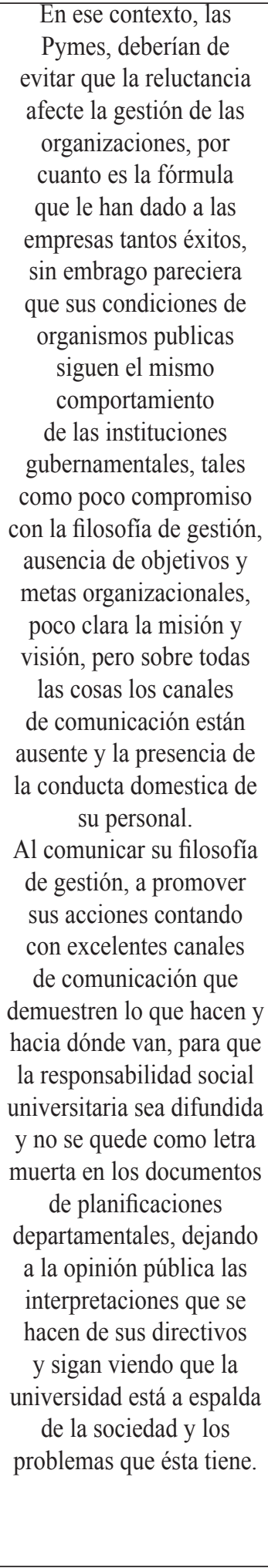 \\
\hline
\end{tabular}




\begin{tabular}{|c|c|c|c|c|c|}
\hline $\begin{array}{c}\text { Estrategias } \\
\text { de valor para } \\
\text { minimizar la } \\
\text { reluctancia en } \\
\text { Pymes. }\end{array}$ & $\begin{array}{c}\text { Seguimiento al } \\
\text { cliente autonomía } \\
\text { Cultura } \\
\text { organizacional } \\
\text { Riesgos } \\
\text { operativos } \\
\text { cubiertos } \\
\text { Personal } \\
\text { contratado } \\
\text { Estrategias de } \\
\text { fidelización } \\
\text { Segmentos de } \\
\text { empleados } \\
\text { Mejor desempeño } \\
\text { Reconocimientos } \\
\text { económicos } \\
\text { Planes de } \\
\text { formación } \\
\text { Sistema de } \\
\text { outplacement } \\
\text { Plataformas } \\
\text { Tecnológicas } \\
\text { Beneficios } \\
\text { adicionales }\end{array}$ & $\begin{array}{c}\text { Robbins (2014). } \\
\text { Gordon (2004). } \\
\text { Fundación } \\
\text { Confemetal } \\
(2006)\end{array}$ & 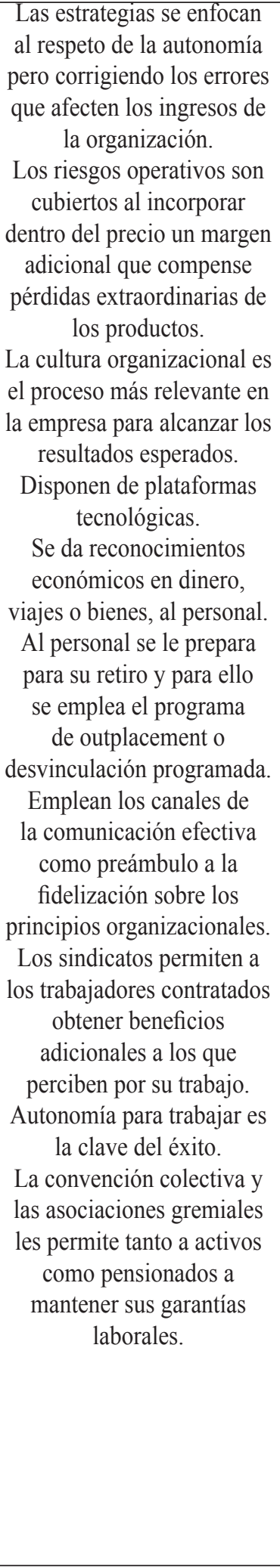 & $\begin{array}{l}\text { Reconocimiento } \\
\text { Estrategias de } \\
\text { fidelización } \\
\text { Sistema de } \\
\text { outplacement } \\
\text { Plataforma } \\
\text { tecnológica }\end{array}$ & 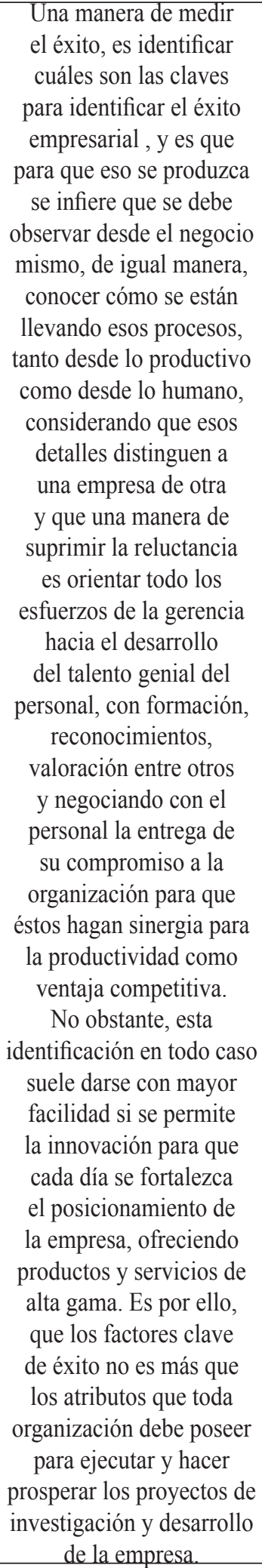 \\
\hline
\end{tabular}

Fuente: Gamboa (2016). 
Diagrama 2. Esquema operativo del Lineamiento Estratégico 1

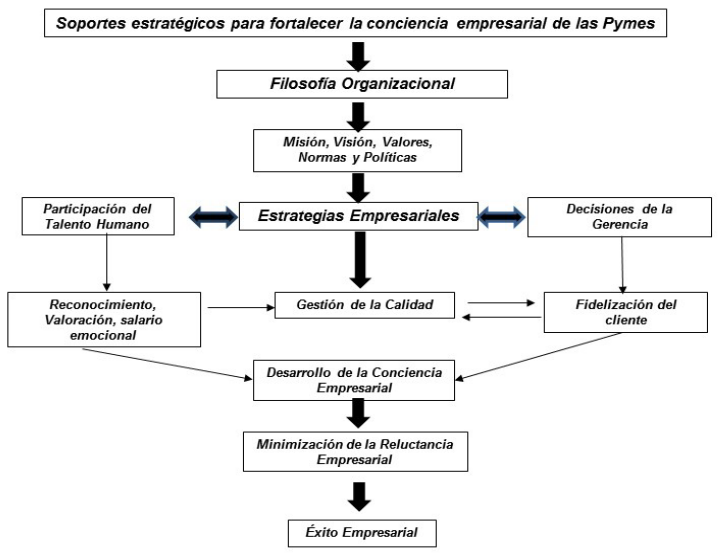

Fuente: Elaboración propia (2016).

Diagrama 3. Esquema operativo del Lineamiento Estratégico 2

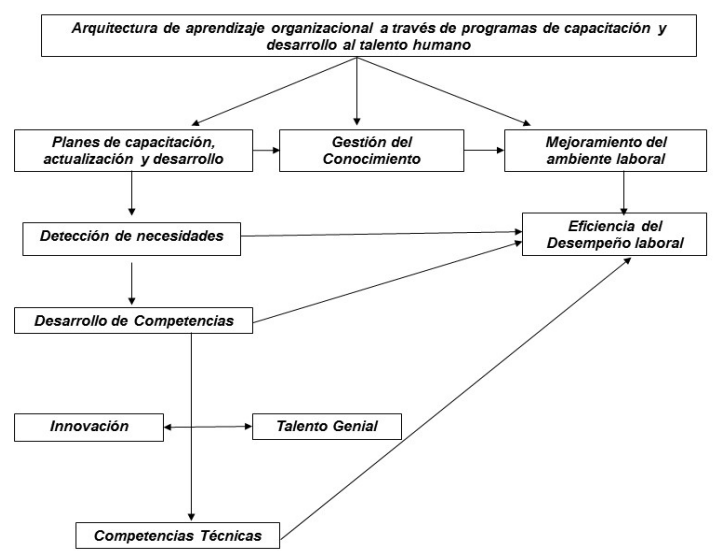

Fuente: Elaboración propia (2016). 
Diagrama 4. Esquema operativo del Lineamiento Estratégico 3

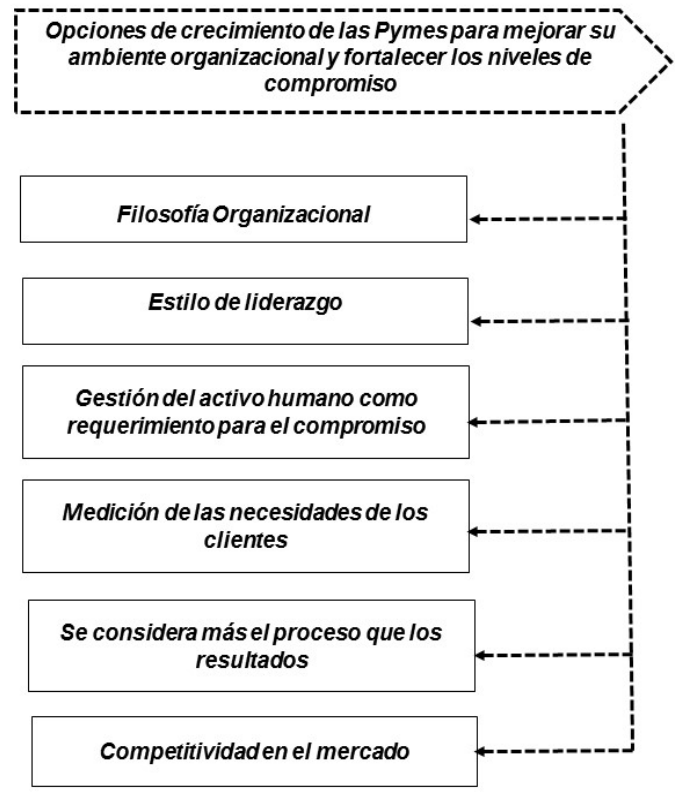

Fuente: Elaboración propia (2016).

Diagrama 5. Esquema operativo del Lineamiento Estratégico 4

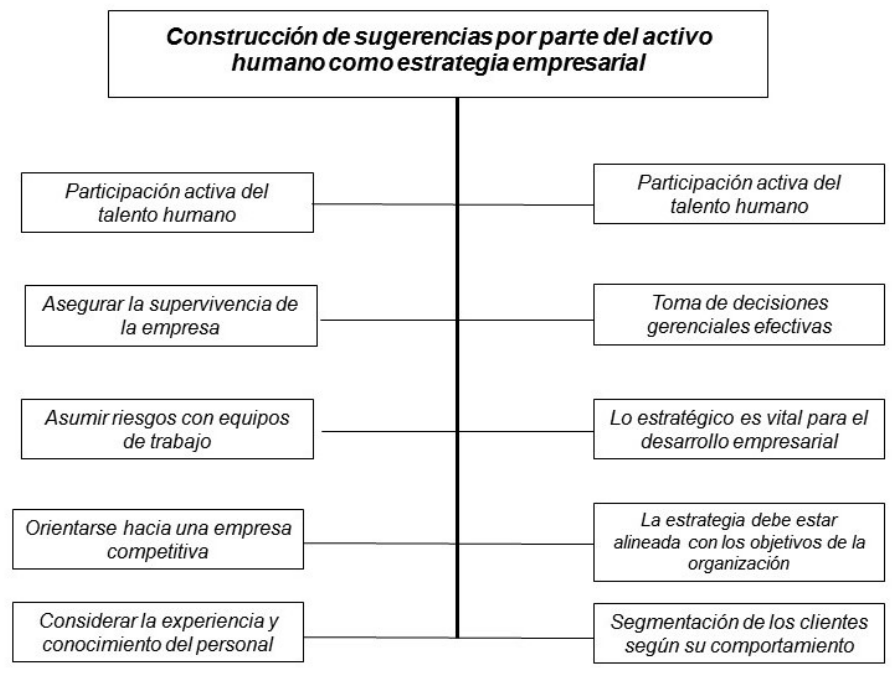

Fuente: Elaboración propia (2016).

\section{Conclusiones}

En Colombia las Pymes se encuentran en franco crecimiento, y es que, como economía emergente, está dando pasos muy apresurados al desarrollo industrial y social, de allí que el surgimiento de nuevas empre- 
sas es la razón de ser del actual gobierno. Lo importante de todo esto es que se están preparando para el duro escenario de la competitividad con visión gerencial y sobre todo apostándole al talento humano que trabajan para ellas.Por tanto, se presentan a continuación las consideraciones finales de esta investigación, que se orientaron de manera que, diera respuesta a cada uno de los propósitos planteados.

En cuanto al propósito central formulado como analizar la gestión del éxito organizacional como estrategia de valor para minimizar la reluctancia en Pymes de Neiva, Departamento del Huila Colombia se puede concluir que el éxito de una empresa en crecimiento debe ser gestionada desde la visión de la filosofía del negocio, por cuanto todo su apalancamiento estratégico está sustentado en las experiencias que le han garantizado su posicionamiento.

Por otro lado, han logrado demostrar en el tiempo que su éxito organizacional se ha apoyado en los valores corporativos, y su talento humano, por cuanto de esta manera, la fatiga o el agotamiento laboral denominado reluctancia, puedan ser minimizados, siempre y cuando se valorice al personal y se considere su participación en las decisiones de la empresa. In embargo hay que considerar que los modelos de gestión de algunas de ellas no les permiten flexibilizar algunas participaciones del personal para la toma de decisiones, sin embargo, se considera su fidelidad y reconocimientos.

Con respecto al propósito derivado que se refiere a identificar los factores clave para la gestión del éxito organizacional en las Pymes, se puede resumir señalando que dentro de esos factores se encuentran sujetos a la capacidad de compromiso del talento humano, donde la autonomía y la participación en la toma de decisiones se considera sumamente importante, sumado a ella la capacitación permanente es condición que puede garantizar el éxito de la empresa, siempre y cuando su filosofía corporativa esté sujeta a la cultura estratégica de la organización.

Por su parte, el propósito derivado que se pretendió describir, se enfoca en los elementos generadores de la posición competitiva global en las Pymes, se puede resumir que la política principal es que entre la orientación así como la atención que presta el personal a los clientes y la flexibilización de precios, productos o servicios, es hacerles participar en los diseños de empaques y valor agregado del servicio, sumado a que su principal acción estratégica es el trabajo en equipo para hacerle frente a la competencia, sumado al plan de carrera que se le presta al personal, asumiendo parte del salario emocional.

Con respecto al propósito derivado sobre las actividades organizacionales que determinan a la reluctancia en las Pymes, se puede concluir que dentro de las estrategias formuladas para minimizar la reluctancia es que para fidelizar y captar a los clientes está el asesoramiento permanente y comprometido, por cuanto su 
satisfacción es lo más importante por el personal, por su parte, los errores lo ven como un aprendizaje lo que permite que generen la gestión de la innovación, otorgándoles mayor soporte a las áreas técnicas. De igual manera, para que el personal se ajuste a la cultura organizacional, se utilizan los valores como mecanismos de soporte de este compromiso.

En referencia a las reconocer las estrategias de valor para minimizar la reluctancia en las Pymes, se van corrigiendo los errores para evitar afectar la rentabilidad, asumiendo los riesgos operativos para alcanzar los resultados esperados. Del mismo modo, se dispone de un perfil tecnológico que es aprovechado para explotar la marca, sumando los reconocimientos económicos al personal tales como viajes, dinero, planes apegados al beneficio de la familia entre otros aspectos, algo muy importante y es la manera de desvincular al trabajador de su tiempo laboral y es la aplicación del outplacement, para darle valor a esos años de esfuerzos, sumado al manejo efectivo de los canales de comunicación efectiva, como marco de la fidelización incluyendo los estándares salariales.

En síntesis, después de analizar las cinco propósitos derivados se puede evidenciar que la gestión del éxito de una organización depende de varios factores fundamentales, como lo es la participación del talento humano, el servicio al clientela manera de gestionar los procesos para fidelizar los clientes, y la manera como se valora y reconoce lo que el personal realiza por la empresa, sin embargo se dan ciertas condiciones que de una manera u otra pudieran frenar parte de ese crecimiento organizacional, y la fórmula para minimizar la reluctancia es apalancarse a la filosofía del negocio, la manera en cómo se fortalece la cultura, sus valores y políticas, apoyado a los programas de formación y capacitación del talento humano.

\section{Referenciada}

Berger, P. Luckmann, T. (1994): La construcción social de la realidad. Buenos Aires. Amorata Editores. Carvajal, R. (2002). De lo humano y lo humanizable en las relaciones de subordinación. Filosofía, historia y sociología del Humanismo en la empresa. Universidad del Valle, Facultad de Ciencias de la Administración.

CONPES (2012), Consejo Nacional de Política Económica y Social República de Colombia Departamento Nacional de Planeación. Secretaría de Transparencia. Presidencia de La República. Imprenta Nacional De Colombia. Noviembre, 2012.

CONPES (2013), Consejo Nacional de Política Económica y Social República de Colombia Departamento Nacional de Planeación. Secretaría de Transparencia. Presidencia de La República. Imprenta Nacional De Colombia. Diciembre, 2013.

El tiempo, enero 19 de 2015 
Fantova, F. (2005). Nuevos modelos en gestión social: calidad y excelencia en las organizaciones sociales. Para la participación prevista en el Congreso Internacional de Calidad de Vida de Personas con Discapacidad, organizado en Quito por la Fundación General Ecuatoriana (17-21 de octubre de 2005).

Flick, U. (2012). Introducción a la investigación cualitativa. Tercera Edición. Editorial Morata.

Gutiérrez, M. (2012). Administrar para la calidad. Segunda edición. Editorial Limusa. México D.F.

Münch, L. (2007). Más allá de la excelencia y de la calidad. Editorial Trillas.

Peiffer, V. (2001). Pensamiento positivo. Editorial: Robin Book (2001). ISBN 10: 8479275529. ISBN 13: 9788479275525.

Peters, T. y Waterman, R. (1986), En busca de la excelencia, Barcelona, Editorial Folio.

Porter, M. (1980): “Competitive Strategy”, Free Press, Nueva York.Rohman (2014)

Seguí. E. (2007). Las singularidades del capital humano de las cooperativas como elementos caracterizadores de su capital intelectual. CIRIEC - España. Revista de economía pública, social y cooperativa, ISSN 0213-8093, Nº 57, 2007 (Ejemplar dedicado a: Transparencia, información y gestión eficiente en la economía social), págs. 93-116.

Strauss, A y Corbin, J. (2002). Bases de la investigación cualitativa. Técnicas y procedimientos para desarrollar la teoría fundamentada. Medellín, Editorial Universidad de Antioquia.

Summers, D. (2006). Administración de la Calidad. Primera Edición. Pearson Educación. México. 